\title{
APLICACIÓN DEL M-LEARNING EN EL AULA DE PRIMARIA: EXPERIENCIA PRÁCTICA Y PROPUESTA DE FORMACIÓN PARA DOCENTES
}

\section{APPLICATION OF M-LEARNING IN THE ELEMENTARY CLASSROOM: PRACTICAL EXPERIENCE AND TRAINING GIVEN TO TEACHERS}

\author{
Dolores Madrid Vivar ; Imadrid@uma.es \\ Ma Jose Mayorga Fernández; mjmayorga@uma.es \\ Fabián Núñez Avilés; vaypase@hotmail.com \\ Facultad de Ciencias de la Educación. \\ Universidad de Málaga
}

\section{RESUMEN:}

Este artículo presenta una experiencia realizada con telefonía móvil. La experiencia ha sido llevada a cabo con alumnado de tercer ciclo de primaria. Después de los resultados obtenidos, consideramos imprescindible hacer una propuesta de formación para el profesorado. En concreto, hemos diseñado un taller cuyo objetivo es utilizar las tecnologías de la información y la comunicación para aumentar la motivación del alumnado y mejorar, en consecuencia, el proceso de enseñanza-aprendizaje.

PALABRAS CLAVE: Competencia digital, formación docente, NNTT, web 2.0, códigos QR, aplicación didáctica.

\section{ABSTRACT:}

This article presents an experience made with mobile phones. The experience has been carried out with students of primary school. After the results of this experience, we consider it essential to propose training for institutions or bodies who are responsible for initial training or teachers. Specifically, we have designed a workshop which aims to use information technology and communication to increase student motivation and improve, therefore, the teaching-learning process.

KEY WORDS: Digital competence, teacher training, ICT, web 2.0, QR codes, didactic application. 


\section{INTRODUCCIÓN}

Las tecnologías de la información y de la comunicación están teniendo consecuencias en la sociedad en general y sobre el ámbito educativo, en particular. Últimos estudios como los de Williams (2008), Reansbottom y Toth (2008), Sultan (2010) y Prensky (2010) reflexionan sobre cómo la juventud de hoy en día convive en una sociedad digital donde el fenómeno social de internet se ha convertido en un medio de transformación.

Ya Sevillano (1998) nos advertía que si queríamos acortar la tradicional distancia que separa a la realidad de dentro y fuera de la escuela, la escuela debía responder integrando en sus espacios estas nuevas formas de comunicación, compartiendo símbolos, medios y recursos, en sintonía con la sociedad. No podemos quedarnos impasibles ante este fenómeno y esperar a que los alumnos tengan que conocer las posibilidades de estas tecnologías por su cuenta fuera del contexto escolar. No haremos más que agravar la distancia que separa a la escuela de la sociedad en la que se circunscribe, al enfrentar los conocimientos que se imparten en la escuela con instrumentos tradicionales con los que se propagan fuera de ella a través de medios mucho más poderosos y atrayentes.

Una mayor dotación de recursos tecnológicos y una mejor explotación educativa de los mismos facilitaría la disminución de las diferencias entre sociedad y escuela, las formas de aprender se complementarían y se compensarían, en parte, las desventajas de los colectivos desfavorecidos en el acceso al uso y disfrute de las posibilidades tecnológicas que ofrece esta época. La escuela cumpliría su función compensadora.

Conviene insistir en que el caballo de batalla no está sólo en disponer en los centros de este u otro material tecnológico, sino en la correcta utilización del mismo que se deriva de su integración organizativa y didáctica.

Por otra parte, la investigación de cómo aplicar TIC al campo educativo ha tenido, y sigue teniendo, mucho que decir (Vidal, 2006). El aumento de trabajos es un hecho patente.

Ya se ha constatado que el uso o incorporación de las TIC en el aula puede ser una buena oportunidad para irse planteando cuestiones necesarias sobre la enseñanza y el aprendizaje en los centros educativos del siglo XXI (Adell, 2010a; 2010b; Domingo \& Fuentes, 2010; Domingo y Marques, 2011). Las líneas de investigación actuales están centrándose en las posibilidades de Internet como entorno y medio en donde se pueden desarrollar los procesos de enseñanza-aprendizaje (Beltrán, 2001; Castells y Díaz de Isla, 2001; Gallego, 2001).

Las aportaciones de estos estudios, junto a los avances tecnológicos están abriendo las puertas a nuevas formas y oportunidades de acceso al proceso de enseñanza-aprendizaje, fundamentalmente porque cada vez contamos con mayor número de herramientas y aplicaciones web 2.0 facilitadoras de estos procesos, pudiendo hacer los contenidos más significativos.

El término Web 2.0 está asociado a aplicaciones web que facilitan el compartir información, la interoperabilidad, el diseño centrado en el usuario y la colaboración en la World Wide Web. Un sitio Web 2.0 permite a sus usuarios interactuar y colaborar entre sí como creadores de contenido generado por usuarios en una comunidad virtual, a diferencia de 
sitios web donde los usuarios se limitan a la observación pasiva de los contenidos que se ha creado para ellos. En la siguiente imagen se muestran algunas de las aplicaciones web 2.0.

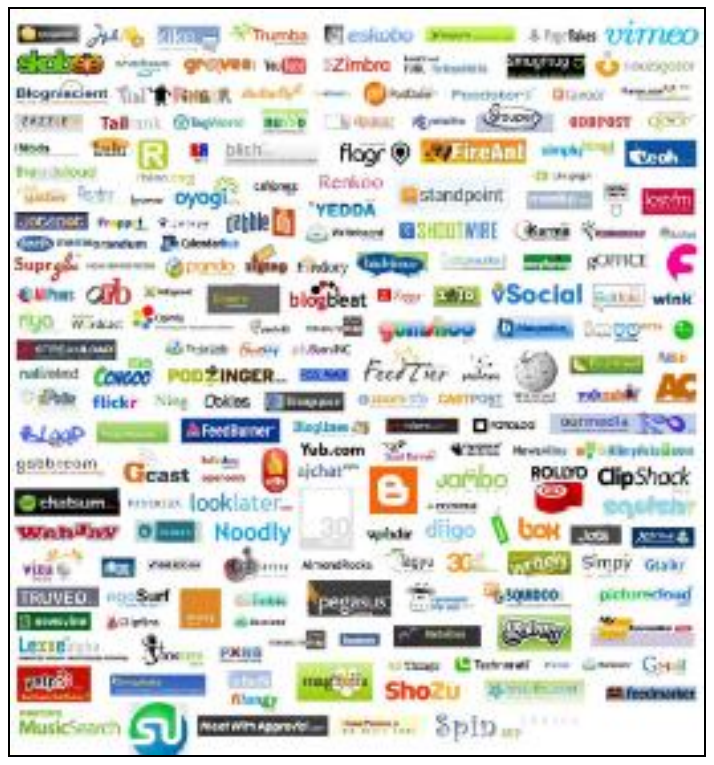

Imagen 1: Escuela Web 2.0

La vida en las aulas no puede estar desconectada de la vida real, de entre todas las posibilidades nuestro artículo presenta una experiencia realizada con telefonía móvil, concretamente con smarphones, hablamos por tanto de lo que se ha denominado ya mobile-learning (ML).

\section{EL M-LEARNING}

De forma paralela al desarrollo de las tecnologías móviles, y la telefonía móvil en particular, ha surgido una corriente, relativamente reciente (aproximadamente una década) de investigaciones interesadas en estudiar qué implicaciones y aplicaciones de dispositivos electrónicos móviles en el campo educativo: el Mobile-learning.

No hay unanimidad sobre qué es el ML (Caudill 2007). Winters (2006) señalaba tres tendencias en relación a la definición de ML: tecnocéntrica; evolutiva a partir del e-Learning; y centrada en el estudiante y su contexto.

En los inicios del ML, los estudiosos lo habían definido como la aplicación de los dispositivos electrónicos móviles para fines educativos (tales como una PDA, reproductores de MP3, un smarthphone, un iPod, Netbook, Tablet PC o un teléfono móvil). Por tanto tecnología móvil y aprendizaje aparecían indisolublemente unidos (Traxler 2005; Parsons y Ryu, 2006).

Otros, sin embargo, consideraron el ML como una evolución del e-learning e incorporaron el valor de la ubicuidad total del aprendizaje, es decir, poder aprender en cualquier momento y cualquier lugar (Quinn, 2000). Mientras que el e-learning permite una gran flexibilidad respecto a los espacios y los tiempos, pero dependiente de la necesidad física de un ordenador y una conexión a Internet, en el ML esta dependencia desaparece. No importa cuando sea o donde estemos, siempre podremos acceder a los contenidos de aprendizaje. 
Sin embargo una tercera tendencia considera el ML no como una evolución del e-learning sino como una nueva modalidad de enseñanza a distancia y enfoca la definición hacia el estudiante en dos aspectos: su movilidad y su contexto de aprendizaje. Este concepto de ML como aprendizaje ubicuo ligado a la movilidad y el contexto de aprendizaje del alumno facilitado por las tecnologías móviles, ha servido para construir las bases para la primera teoría acerca del ML.

Nuestro trabajo es una experiencia en $\mathrm{ML}$, que podríamos situar en la primera tendencia.

Si durante muchos años, el debate sobre el uso o no de los teléfonos móviles en los centros educativos se limitaba a la posibilidad de permitir su utilización o no y la medida que se toma habitualmente para evitar el uso de los móviles es su retirada, las cosas están empezando a cambiar, como apunta Hernández (2012). El debate ha comenzado a reabrirse, no exento de polémica. Son muchos los docentes que se muestran temerosos ante su uso en el aula, desconocedores de sus posibilidades o reticentes a incorporar nuevas herramientas. En muchas ocasiones, se desconocen las posibilidades de uso de los teléfonos inteligentes o smarthphones, que han pasado de ser meros elementos de comunicación a ser unidades tecnológicas de última generación capaces de generar contenidos que pueden ser compartidos en cualquier medio, lugar y momento. Los smarthphones son ordenadores que incluso los superan por sus aplicaciones y capacidad para poder usarse de manera inmediata y en cualquier lugar.

A pesar de estos datos, nuestra realidad educativa muestra una perspectiva completamente distinta y contradictoria, contrapuesta totalmente a la realidad social. Y se manifiesta en la gran resistencia a la integración de los teléfonos móviles como una herramienta educativa más en el contexto escolar. Creemos que tres son las grandes barreras para esta integración educativa: en primer lugar las administraciones educativas, en todos los niveles, han dictado directrices para la restricción o total prohibición de los móviles en los centros educativos. En segundo lugar, el alumnado no tiene conciencia de que el móvil pueda ser utilizado con fines educativos, lo consideran solo como un medio lúdico. En tercer lugar, el profesorado, en general, desconoce la potencialidad educativa de estos dispositivos que contemplan como un elemento disruptivo o de intromisión en el aula. Sin embargo, no podemos dar la espalda a la realidad cotidiana: el teléfono móvil constituye un elemento integrante de nuestra vida cotidiana y diríamos que hasta imprescindible en la de nuestro alumnado (Brazuelo y Cacheiro, 2010).

Esta última barrera es la que intentamos eliminar presentado nuestra experiencia práctica, sus resultados y una propuesta para la formación de los docentes.

\section{LA EXPERIENCIA Y SUS RESULTADOS}

La experiencia que mostramos en este apartado fue llevada a cabo con el alumnado de tercer ciclo de primaria del CEIP Mare Nostrum de Torrox (Málaga). Dentro de la programación de aula, se propusieron una serie de actividades complementarias en el área de Conocimiento del Medio con el objetivo de motivar al alumnado y de reforzar y ampliar los contenidos curriculares, además de trabajar de manera transversal las competencias de aprender a aprender y la tecnológica. De esta forma, en cada trimestre el profesor-tutor elaboró un juego de preguntas a modo de enigma para que el alumnado lo resolviera. Se 
necesitó la ayuda de algunos padres y madres de alumnos y alumnas que utilizaban la tecnología de los móviles smartphone con conexión de datos.

Para la elaboración del enigma, se buscaron preguntas que provocaran una actitud investigadora del alumnado, por ejemplo, ¿cuándo se llegó a la luna por primera vez? Esto generó de forma inmediata la búsqueda de información en la red por parte del alumnado.

La actividad se desarrolló en las zonas comunes y pista polideportiva del centro escolar. El proceso para la puesta en práctica fue el siguiente:

- 10 Colocación de los códigos QR en zonas visibles, principalmente en el patio exterior y pista polideportiva del colegio. Esta tarea se realizó un día antes de la puesta en práctica de la propia actividad. Cada enigma tenía entre diez y quince preguntas, con el propósito de que éste no fuese ni muy corto ni muy largo de resolver. Hay que tener en cuenta que la propia organización del centro escolar nos limita el tiempo de ejecución que en nuestro caso se prolongó durante dos sesiones o tramos horarios.

- 20 Formación de equipos. Cada equipo estaba compuesto por cinco alumnos/as y el apoyo de un padre o madre para utilizar el lector de códigos QR en el Smartphone. Así se formaron cinco grupos constituidos cada uno por cinco alumnos/as y un padre o madre.

- 3o Resolución del enigma. Cada grupo podían buscar los códigos QR y resolver las preguntas en el orden que quisieran, pero los miembros que lo forman no se podían separar en ningún momento. Cada grupo disponía de uno o varios ultraportátiles de dotación del programa Escuela 2.0. para buscar en Internet las soluciones. Cada grupo anotaba las soluciones para posteriormente resolver el enigma en red.

Para la realizar la evaluación de esta actividad, elaboramos y aplicamos un cuestionario de valoración al profesorado implicado y al alumnado participante. La valoración en cada pregunta se contestaba señalando de 1 (poco) a 5 (mucho). Las cuestiones recogidas en ambos cuestionarios son las siguientes:

A. Cuestionario para el profesorado:

1. ¿Al introducir los temas procuras llamar su atención presentando una información novedosa?

2. Cuando te propones que tus alumnos expongan lo que ya saben sobre un nuevo tema ¿varías el tipo de actividades? Por ejemplo, alternando: diálogos, cuestionarios, análisis de situaciones problemas, explicando lo que les gustaría saber, "bombardeo" de palabras que puedan relacionar con el tema,...

3. ¿Relacionas los temas de actualidad o de su interés con los contenidos escolares?

B. Cuestionario para el alumnado:

1. ¿Han surgido discusiones con tus compañeros durante el desarrollo de la actividad?

2. ¿Te has separado del grupo en algún momento?

3. ¿Has consultado Internet para resolver las preguntas?

4. ¿Crees que has aprendido otros contenidos relacionados con la asignatura?

5. ¿Te ha gustado el juego que has realizado?

6. ¿Te gustaría realizar este tipo de juegos con otros contenidos de la asignatura?

7. ¿Te parece bien que algunos padres y madres colaboren con esta actividad? 
A continuación se incluyen los principales resultados:

Una vez analizado el cuestionario del profesorado, se aprecia que dicho profesorado al plantear las cuestiones del enigma procura que sean temas que capte la atención del 100\% de su alumnado. Las preguntas son variadas, pero no excesivamente para no dispersar dicha atención. En gran medida se suele relacionar el contenido escolar con temas actuales y de interés para el alumnado.

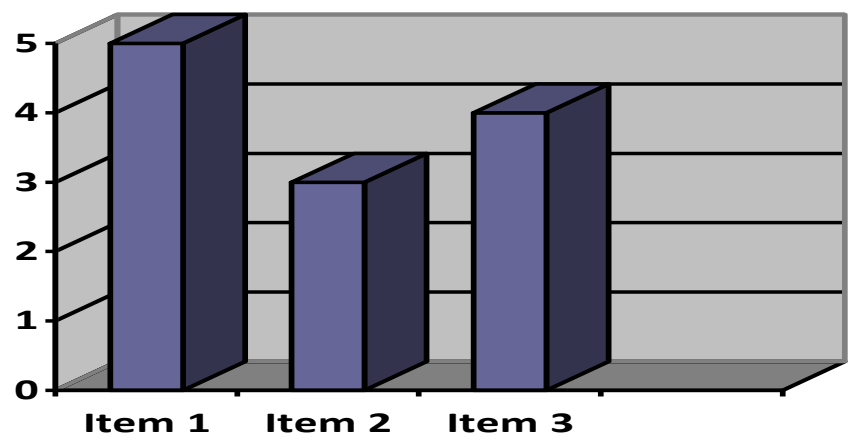

\section{$\square$ Moda}

Gráfica 1: resultado cuestionario profesores

Al observar los resultados del cuestionario del alumnado se aprecia que la valoración ha sido muy positiva, como se muestra en la gráfica 2 .

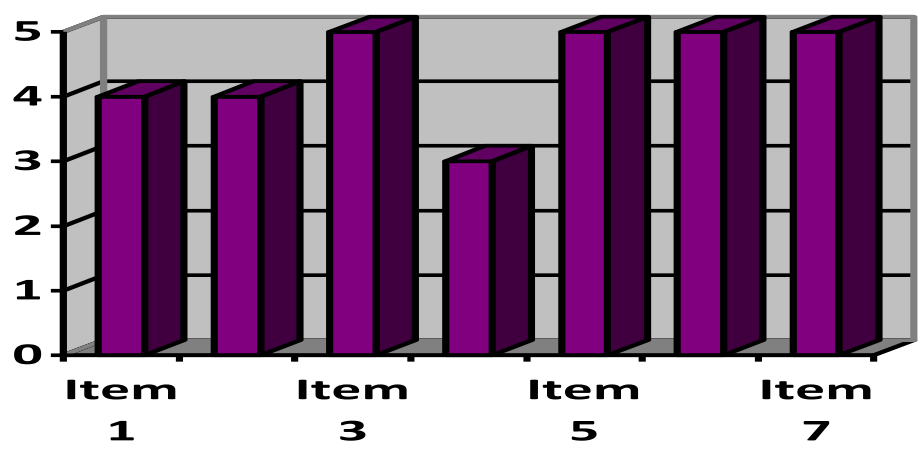

$\square$ Moda

Gráfico 2: resultado cuestionario alumnado

La actividad no ha generado discusiones entre el alumnado, han trabajado constantemente en equipo, consultado internet para resolver las cuestiones. El ítem menos valorado $(\operatorname{moda}=3)$ hace referencia a que el alumnado no está muy seguro de haber aprendido otros contenidos relacionados con la asignatura, pero a pesar de ello, les ha gustado realizar este juego, y les agradaría seguir realizando actividades de estas características con el apoyo de sus padres.

En definitiva, los resultados muestran que la experiencia ha sido muy positiva, poniendo de manifiesto que:

- Se incrementa la cooperación entre los miembros del grupo. 
- Se ven implicados otros miembros de la comunidad educativa: padres y madres del alumnado.

- Se potencia una actitud investigadora.

- Se amplían y refuerzan contenidos del área curricular.

- Se observa mayor implicación y motivación del alumnado.

\section{PROPUESTA DE FORMACIÓN}

La formación y la actualización del profesorado, es el camino adecuado para conseguir la incorporación de los recursos tecnológicos al terreno educativo, es una idea compartida por todos los expertos en este ámbito de estudio.

Sin embargo, las investigaciones también constatan como muchos de estos profesionales de la educación no han desarrollado una actitud favorable hacia ella. Por ejemplo Aznar, Fernández e Hinojo (2003), que diseñaron una escala tipo likert para el estudio de las actitudes respecto a las TIC, interpretan que esto puede ser una de las razones por las que no las utilicen en el aula, lo que conlleva, a una falta de aprovechamiento de lo que estos recursos pueden aportar tanto a su trabajo como al aprendizaje del alumnado.

Ortega y Fuentes (2003) afirman en un interesante artículo donde abordan el tema de la tecnofobia docente, que un alto porcentaje de los docentes entrevistados declara que desconocen la oferta de formación continua disponible; durante el ejercicio profesional la mayor parte de los docentes manifiesta haber conseguido mejorar sus conocimientos mediante la realización de cursos ofertados por los Centros de Profesorado; la formación recibida durante el ejercicio profesional se considera, en general, alejada de las demandas de la realidad escolar.

Sin embargo, el docente es un "eslabón" fundamental en la "cadena" de nuestro sistema educativo (Escudero, 2000; Mateo, 2000); por tanto, la formación docente en recursos tecnológicos es una premisa fundamental, y concretamente la aplicación de las tecnologías de la información y comunicación a las tareas escolares en cada una de las áreas que forman el currículum de Educación Infantil, Primaria y Secundaria, para alcanzar la optimización y la eficacia en los procesos de enseñanza-aprendizaje.

Por tanto, entendemos como indispensable, de cara a la mejora de la profesionalización del docente, que se dote al profesorado de los recursos y habilidades necesarias para el dominio de los nuevos soportes tecnológicos (Sánchez, Boix y Jurado, 2009), la propuesta de un taller nos ha parecido una acertada opción.

Después de los resultados obtenidos en la experiencia, consideramos imprescindible hacer una propuesta de formación para aquellas instituciones u organismos que tengan la responsabilidad de la formación inicial o de la permanente. Hemos diseñado un taller estructurado en cinco partes claramente diferenciadas, de manera sintetizada lo presentamos a continuación. 


\begin{tabular}{|c|c|}
\hline $\begin{array}{l}\text { PARTE 1: Búsqueda } \\
\text { de información }\end{array}$ & $\begin{array}{l}\text { Búsqueda de información para elaborar las preguntas que forman el enigma. } \\
\text { Las preguntas pueden tener diferente formato: } \\
\text { - Texto, como por ejemplo “¿cómo se llamaba la persona que piso por } \\
\text { primera vez la luna?". } \\
\text { - Imagen, se trata de contestar una pregunta referida a una imagen } \\
\text { mostrada, como por ejemplo "¿quién es el autor de la obra } \\
\text { pictórica?". } \\
\text { - Vídeo, cuando la pregunta se resuelve después de visualizar el vídeo, } \\
\text { - ¿¿quién es el personaje famoso que aparece en el vídeo?" } \\
\text { Música, la pregunta se refiere a un fragmento musical o sonoro, por } \\
\text { ejemplo “¿quién es el autor de la sinfonía?". }\end{array}$ \\
\hline $\begin{array}{l}\text { PARTE 2: Utilizar } \\
\text { herramientas de } \\
\text { autor o aplicaciones } \\
\text { web } 2.0\end{array}$ & $\begin{array}{l}\text { Utilizar herramientas de autor o aplicaciones web } 2.0 \text { para elaborar el juego } \\
\text { de preguntas o enigma en formato digital. } \\
\text { - Aplicaciones o servicios web 2.0: www.educaplay.com Para crear y } \\
\text { compartir nuestras propias actividades educativas o diseñadas por } \\
\text { otros usuarios. } \\
\text { Herramientas de autor offline: Existe una gran variedad de } \\
\text { herramientas para la creación de contenidos digitales: Hotpotatoes, } \\
\text { JClic, Ardora, Lim, Cuadernia, etc. En este caso nos hemos decantado } \\
\text { por LIM: www.educalim.com. Este sistema es un entorno para la } \\
\text { creación de materiales educativos, formado por un editor de } \\
\text { actividades (EdiLim), un visualizador (LIM) y un archivo en formato } \\
\text { XML (libro) que define las propiedades del libro y las páginas que lo } \\
\text { componen. }\end{array}$ \\
\hline $\begin{array}{l}\text { PARTE 3: Juego on- } \\
\text { line }\end{array}$ & $\begin{array}{l}\text { Consistirá en poner online el juego de preguntas creado con LIM, para que } \\
\text { esté disponible para toda la comunidad educativa. Debemos subir los archivos } \\
\text { creados con LIM a la red. Para este fin, utilizamos un disco duro virtual que nos } \\
\text { ofrece algunas de los siguientes compañías: } \\
\text { - Windows Live SkyDrive (inicialmente Windows Live Folders) SkyDrive } \\
\text { permite a los usuarios subir archivos de un ordenador y almacenarlos } \\
\text { en línea (nube), y acceder a ellos desde un navegador web. El servicio } \\
\text { utiliza Windows Live ID para controlar el acceso a los archivos del } \\
\text { usuario, y les permite mantener la confidencialidad de los archivos, } \\
\text { compartir con contactos o compartirlos con el público en general. Los } \\
\text { archivos que se comparten públicamente no requieren una cuenta de } \\
\text { Windows Live ID para acceder. } \\
\text { Dropbox es un servicio de alojamiento de archivos multiplataforma en } \\
\text { la nube, operado por la compañía Dropbox. El servicio permite a los } \\
\text { usuarios almacenar y sincronizar archivos en línea y entre } \\
\text { ordenadores, así como compartir archivos y carpetas con otros. Esto } \\
\text { nos permite disponer de un disco duro o carpeta virtual de forma } \\
\text { remota y accesible desde cualquier ordenador en el mundo. } \\
\text { Para comenzar, debemos de abrir una cuenta para disponer de un } \\
\text { espacio de almacenamiento gratuito. Si optamos por Dropbox, tenemos la } \\
\text { posibilidad de gestionar la subida de archivos directamente vía web o } \\
\text { descargarnos la aplicación en nuestro ordenador. En tal caso, se nos crearáa } \\
\text { una carpeta llamada "dropbox" que estará sincronizada con nuestro disco } \\
\text { duro virtual en la web de } 2 \text { Gb de almacenamiento gratuito. } \\
\text { Llegado a este punto, debemos saber que cualquier objeto en Internet } \\
\text { (imagen, vídeo, texto, página web...) tiene una dirección específica. Por tanto, } \\
\text { nuestro enigma tendrá una dirección web propia cuando ya está alojado en } \\
\text { nuestro disco duro virtual. Dropbox nos dará la dirección html que tiene } \\
\text { nuestra página web. }\end{array}$ \\
\hline
\end{tabular}




\begin{tabular}{|c|c|}
\hline $\begin{array}{l}\text { PARTE 4: Creación } \\
\text { de códigos QR para } \\
\text { resolver los } \\
\text { enigmas a través de } \\
\text { móviles } \\
\text { Smarphone. }\end{array}$ & $\begin{array}{l}\text { Un código QR es un sistema que permite almacenar información en una } \\
\text { especie de código de barras de última generación. } \\
\text { Con la ayuda de un móvil y un lector de códigos podemos recuperar esta } \\
\text { información tan solo con apuntar la cámara hacia el código QR. En unos } \\
\text { segundos aparecerá la información en la pantalla: una imagen, un vídeo, una } \\
\text { página web, etc.). } \\
\text { Para aplicar los códigos QR a nuestro juego de preguntas, tenemos que } \\
\text { almacenar en el disco virtual las imágenes, vídeos o preguntas texto de } \\
\text { nuestro enigma y enlazarlas con un código QR. Para ello, tenemos en la web } \\
\text { servicios gratuitos: } \\
\text { - Opción 1: http://www.qrcode.es/es/generador-qr-code/ } \\
\text { - Opción 2: http://www.qrcode.es/es/generador-qr-code/ } \\
\text { - Opción 3: http://qrcode.kaywa.com/ } \\
\text { Simplemente copiamos la dirección de nuestro objeto en el generador y éste } \\
\text { nos dará la imagen del código QR para descargarla en nuestro ordenador o } \\
\text { imprimirla. }\end{array}$ \\
\hline $\begin{array}{l}\text { PARTE 5: Aplicación } \\
\text { Didáctica. }\end{array}$ & $\begin{array}{l}\text { Una vez que tenemos el enigma elaborado y los códigos QR impresos en papel, } \\
\text { tenemos diferentes estrategias en la práctica para su aplicación didáctica: } \\
\text { a. Resolver el enigma en red sin utilizar la tecnología de los Smarphone. A } \\
\text { partir de una dirección web resolveremos el enigma. } \\
\text { b. Resolver el enigma en red utilizando la tecnología de los Smarphone. Esta } \\
\text { opción es la más enriquecedora con dinámica de grupo para resolver el juego } \\
\text { de preguntas. Se formarán tantos grupos como preguntas tenga el enigma y } \\
\text { cada uno se encargará de resolver una de ellas. Cada grupo tendrá, al menos, } \\
\text { un Smarphone con lector de códigos QR. Se dará un tiempo determinado para } \\
\text { que cada grupo se traslade al lugar donde se encuentra su código QR y } \\
\text { resuelva la pregunta. Cuando el tiempo establecido se haya cumplido, } \\
\text { resolveremos el enigma que se encuentra alojado en dropbox y cada grupo } \\
\text { dará su respuesta. } \\
\text { Para conseguir mayor motivación de los participantes, la resolución del enigma } \\
\text { puede ofrecer un premio o tesoro que en función de nuestros objetivos puede } \\
\text { consistir en: puntos positivos, dar a conocer una noticia importante, } \\
\text { caramelos, chocolatinas, etc. }\end{array}$ \\
\hline
\end{tabular}

Nuestro propósito es dar los pasos necesarios para crear un juego de preguntas o enigma adaptable a cualquier nivel o área curricular. El objetivo de este taller es utilizar las tecnologías de la información y la comunicación para aumentar la motivación del alumnado y mejorar, en consecuencia, el proceso de enseñanza-aprendizaje.

\section{DISCUSIÓN}

Siguiendo la idea de Domingo y Marqués (2011), consideramos que nuestra propuesta ofrece oportunidades para que el profesorado trabaje conjuntamente con expertos, lo que va a favorecer e incentivar probar nuevas estrategias didácticas acordes con nuestros tiempos. Una vez realizado el taller y haber puesto en práctica los contenidos, seria imprescindible organizar un encuentro donde intercambiar las experiencias realizadas $y$ reflexionar sobre las mismas. 
Cada vez se abre más paso la consideración del docente como un mediador del aprendizaje, por ello, desde el ámbito universitario apostamos por una amplia formación en competencia digital. Nuestro alumnado de hoy son el profesorado del mañana. Profesorado que debe tener una actitud positiva hacia las TICs, debe conocer el uso de dichas tecnologías en su área de conocimiento, utilizarlas con destreza e integrarlas en la planificación del currículum, siendo imprescindible plantear talleres como el que presentamos en este trabajo para alcanzar la competencia digital.

Debemos establecer métodos para regular su uso, ponerlos al servicio del aprendizaje y sacarles el máximo rendimiento.

\section{REFERENCIAS BIBLIOGRÁFICAS}

ADELL, J. (2010a). Web 2.0 y Escuela 2.0. DIM-UAB, 16. (http://dim.pangea.org/revistaDIM16/revistanew.htm) (04-09-2010).

ALONSO, M. Y MATILLA, L. (1990). Imágenes en acción: Análisis y práctica de la expresión audiovisual en la escuela activa. Madrid:, Akal.

AZNAR, I.; FERNÁNDEZ, F. E HINOJO, F.J. (2003). Formación docente y TIC: elaboración de un instrumento de evaluación de actitudes profesionales. Etic@net, (http://www.ugr.es/ sevimeco/revistaeticanet/Numero2/Articulos/ActitudesFormaci ondocenteenTIC[1].pdf) (02-10-2012)

BAUTISTA GARCÍA-VERA, A. (1994). Las nuevas tecnologías en la capacitación docente. Madrid: Visor.

BELTRÁN, (2001). La Nueva Pedagogía a través de Internet. (http://www.isabelperez.com/webquest/webquestppt/sld002.htm) (17/10/12).

BLESA, J.A. (2002). Aulas autosuficientes. (http://roble.pntic.mec.es/ jblesa/autosufi.htm) $(09 / 09 / 12)$.

BRAZUELO, F. Y CACHEIRO, M.L. (2010). Diseño de páginas web educativas para teléfonos móviles. EDUTEC, Revista Electrónica de Tecnología Educativa, 32. http://edutec.rediris.es/Revelec2/revelec32/diseño web educativas telefonos mov iles.html (20/10/12).

CABERO, J. (2001). Las tecnologías de la información y comunicación en la Universidad. Sevilla: MAD.

CASTELLS Y DÍAZ DE ISLA (2001). Difusión y uso de internet en Cataluña y España. http://www.uoc.edu/in3/dt/20011/index.html (21/10/12).

CAUDILL, J. G. (2007). The growth of m-learning and the growth of mobile computing: Parallel developments. International Review of Research in Open and Distance Learning, 8, 2. 
DOMINGO, M. Y FUENTES, M. (2010). Innovación educativa: Experimentar con las TIC y reflexionar sobre su uso. Píxel-Bit, 36;171-180.

DOMINGO, M. Y MARQUES, P. (2011). Aulas 2.0 y uso de las TIC en la práctica docente. Comunicar, 37,XIX, 169-175.

ESCUDERO, T. (2000). La evaluación y mejora de la enseñanza en la universidad: otra perspectiva. Revista de Investigación Educativa, 18 (2), 405-416.

GALLEGO, D. (2001). Evolución y desarrollo de las nuevas tecnologías de la información y la comunicación: consecuencias socioculturales y analfabetismo tecnológico. Congreso internacional sobre Ética en los Contenidos de los Medios de comunicación e internet. Centro de UNESCO Andalucía y Fundación para una Cultura de Paz. Granada, 15,16 y 17 de octubre.

GALLEGO, D Y ALONSO, C. (1999): Multimedia en la web. Madrid: Dykinson.

GALLEGO, D. Y GATICA, N. (2010). La Pizarra Digital. Una ventana al mundo desde las aulas. Madrid: MAD, Eduforma.

HERNÁNDEZ, J. (2012). Móviles y apps. Realmente aportan algo a la Educación. Comunicación y Pedagogía, 259-260,41-46.

MAJO, J.; MARQUÉS, P. (2002). La revolución educativa en la era Internet. Barcelona: CissPraxis (http://www.peremarques.net/libros/revoledu.htm) (12/10/12).

MATEO, J. (2000). La evaluación del profesorado y la gestión de la calidad de la educación. Hacia un modelo comprensivo de evaluación sistemática de la docencia. Revista de investigación educativa, 18 (2), 7, 34.

ORTEGA, J.A. Y FUENTES, J. (2003). La sociedad del conocimiento y la tecnofobia del colectivo docente: implicación desde la formación del profesorado. En Comunicación y Pedagogía, 189, pp. 63-68.

PAVÓN, F. (2001). Educación con Nuevas Tecnologías de la Información y la Comunicación. Sevilla: Kronos.

PARSONS, D. Y RYU, H. (2006). A framework for assessing the quality of mobile learning. (http://www.masey.ac.nz/ hryu/M---learning.pdf ) (10/10/12).

PRENSKY, M. (2010). Teaching digital natives. Partnering fotr real learning. Thousand Oaks. CA: Corwin Press.

Protégeles.com (2005). Seguridad Infantil y Costumbres de los Menores en el Empleo de la Telefonía Móvil. Madrid: Oficina del Defensor del Menor.

QUINN, C. (2000). MLearning: Mobile, Wireless, in your Pocket Learning.(http://www.linezine.com/2.1/features/cammwiyp.htm) (20/10/12).

REANSBOTTOM, B. Y TOTH, C. (2008). The mash-up of web 2.0. technologies the future of podcasting and social networking. Paper presented al the internacional conference of the society for information technology and teacher education. Las Vegas: Nevada. 
RODRÍGUEZ DIÉGÜEZ, J.L. (1995): Tecnología educativa: Nuevas Tecnologías Aplicadas a la Educación. Alcoy: Marfil.

SÁNCHEZ, A.; BOIX, J. Y JURADO, J. (2009). La sociedad del conocimiento y las tics: una inmejorable oportunidad para el cambio docente. Píxel Bit, 34, 174-204.

SEVILLANO, Mạ.L. (coord.) (1998). Nuevas tecnologías, medios de comunicación y educación. Madrid: CCS.

SEVILLANO, Mạ.L. (Coord.) (2002) 2a ed.: Nuevas Tecnologías, Medios de Comunicación y Educación. Madrid: CCS.

SILVA, S. (2005). Informática educativa. Usos y aplicaciones de las Nuevas Tecnologías en el aula. Vigo: Ideas propias.

SULTAN, N. (2010). Cloud computing for education: a new dawn. Internacional Journal of information management, 30 (2), 109-116.

TRAXLER, J.(2005). MobileLearning: It's here but what is it?

(http://www2.warwick.ac.uk/services/cap/resources/interactions/archive/issue25/traxler/) (20/10/12).

VIDAL, M.P. (2006). Investigación de las TIC en educación. Revista latinoamericana de Tecnología Educativa, 5 (2), 539-552.

WILLIAMS, B. (2008). Tomorrow will not be like today: literacy and identity in a world of multiliteracies. Journal of adolescent \& adult literacy, 51 (8). 682

WINTERS, N. (2006). What is mobile learning? In M. Sharples (ed.), Big Issues in Mobile Learning: Report of a workshop by the Kaleidoscope Network of Excellence Mobile Learning Initiative. Nottingham: University of Nottingham.

\section{Para citar este artículo:}

MADRID, D., MAYORGA, M. J. \& NÚÑEZ, F. (2013). Aplicación del m-learning en el aula de primaria: Experiencia práctica y propuesta de formación para docentes. EDUTEC, Revista Electrónica de Tecnología Educativa, 45. Recuperado el dd/mm/aa de http://edutec.rediris.es/Revelec2/Revelec45/aplicacion mlearning primaria experiencia fo rmacion docentes.html 